\title{
Effectiveness of nonpharmacological interventions for reducing postpartum fatigue: A systematic review and meta- analysis
}

Jialu Qian

Zhejiang University School of Medicine

Shiwen Sun

Women's Hospital School Of Medicine Zhejiang University

Lu Liu

Zhejiang University School of Medicine

Xiaoyan Yu ( $\nabla$ yuxy@zju.edu.cn )

Women's Hospital School Of Medicine Zhejiang University

\section{Research Article}

Keywords: Fatigue, Postpartum, Nonpharmacological interventions, Systematic review, Meta-analysis

Posted Date: April 23rd, 2021

DOI: https://doi.org/10.21203/rs.3.rs-402893/v1

License: () (1) This work is licensed under a Creative Commons Attribution 4.0 International License. Read Full License 


\section{Abstract}

Background: Postpartum fatigue is the most common issue among postnatal women and it could not only seriously affect the health of mothers but also bring about adverse impacts on their offspring. However, postpartum fatigue is an ongoing research issue but is seldom treated. This systematic review and meta-analysis aims to synthesize nonpharmacological evidence and evaluate the effectiveness of interventions for reducing postpartum fatigue among puerperae.

Methods: The Cochrane Library, PubMed, Embase, Web of Science, PsycINFO, CINAHL and ProQuest databases were searched for papers published from inception until February 2021. Grey literature was searched using OpenGrey. Randomized controlled trials (RCTs) or controlled clinical trials (CCTs) evaluating nonpharmacological interventions for postpartum fatigue reduction were eligible for inclusion. The methodological quality of the included studies was independently assessed by two reviewers using the Cochrane risk-of-bias tool and the risk of bias in nonrandomized studies of interventions. The metaanalysis was conducted using Review Manager 5.3.

Results: Seventeen published clinical trials matched the eligibility criteria for the systematic review, and thirteen studies involving 1686 participants were included in this meta-analysis. The results of the meta-analysis revealed that exercise (SMD $=-1.74,95 \% \mathrm{Cl}=-2.61$ to -0.88$)$, physical therapy $(\mathrm{SMD}=-0.50,95 \%$ $\mathrm{Cl}=-0.96$ to -0.03$)$ and drinking tea $(\mathrm{MD}=-3.12,95 \% \mathrm{Cl}=-5.44$ to -0.80$)$ resulted in significant improvements in women's postpartum fatigue at postintervention. Drinking tea may have beneficial effects on depression ( $\mathrm{MD}=-2.89,95 \% \mathrm{Cl}=-4.30$ to -1.49$)$. Positive effects of psychoeducational interventions on postpartum fatigue or depression were not observed.

Conclusions: This review provides evidence that exercise, physical therapy and drinking tea are effective nonpharmacological interventions for relieving postpartum fatigue. Detailed instructions for postpartum exercise should be offered to puerperae. Physical therapy could be used in combination to enhance the intervention efficacy. Multiple daily cups of tea may be recommended. Psychoeducational interventions were ineffective for postpartum fatigue, but they could be integrated with the internet or smartphones to improve their effectiveness in the future. Fatigue-related nonpharmacological interventions of psychological outcomes still need to be studied.

\section{Background}

Postpartum fatigue is considered the most common issue that postnatal women confront when they transition to motherhood[1]. Postpartum fatigue is described as feelings of suffocation, exhaustion, and decreases in physical and mental capacity[2]. These symptoms may disturb approximately $64 \%$ of mothers in their postpartum stage[1]. It was reported that $38.8 \%, 27.1 \%$ and $11.4 \%$ of women perceived fatigue at 10 days, 1 month and 3 months after delivery, respectively, which indicates that the influence of postpartum fatigue on puerperae is general and persistent. Negative psychological symptoms (e.g., depression, anxiety and stress), sleep problems and less effective parenting behaviours are closely associated with the severity of postpartum fatigue[3-5]. Importantly, previous research demonstrated that postpartum fatigue could not only seriously affect the maternal health of mothers but also bring about adverse impacts on their offspring[6]. Experiences of fatigue could negatively affect breastmilk production, maternal-infant attachment and interactions[2, 5 , 7], thereby delaying the development of babies[8].

Hence, it is significant to deliberately avert and relieve fatigue during the postpartum period via healthy and effective approaches. In fact, interventions for reducing postpartum fatigue have particular advantages. They are not only important to puerperae' physical relief but also have potential benefits on the improvement of maternal mental health. Cindy-Lee et al.[9] suggested that preventing postpartum depression could start from the perspective of fatigue management. Compared with recommended psychotherapy, fatigue management is less stigmatising and may be an acceptable first step for women to seek assistance and receive treatment for psychological issues[9].

However, postpartum fatigue is an ongoing research issue but is seldom treated. On the positive side, there is rapidly growing interest in this area. Although studies of nonpharmacological interventions conducted in puerperae with the aim of reducing fatigue are accumulating, different interventions may lead to varied effects[10-13]. Previous relevant systematic reviews and meta-analyses have clarified the positive relationship of postpartum fatigue and depression[3], the predictive factors of postpartum fatigue[1] and the effects of exercise on pregnancy and postpartum fatigue[14].

To the best of our knowledge, there is currently no in-depth systematic review and meta-analysis of nonpharmacological interventions specific for postpartum fatigue. Therefore, the aim of this study was to identify existing nonpharmacological interventions offered to postnatal women and to examine their effectiveness for relieving postpartum fatigue.

\section{Methods}

\section{Protocol and registration}

The protocol has been registered in the International Prospective Register of Systematic Reviews (PROSPERO); registration number CRD42021234869.

\section{Study design}

This systematic review and meta-analysis has been reported following the Preferred Reporting Items for Systematic Reviews and Meta-Analysis statement (PRISMA). The PRISMA checklist can be seen in Additional file 1.

\section{Search strategy}

The focus of the review was to examine nonpharmacological interventions for fatigue reduction in postpartum women. Seven electronic databases, including the Cochrane Library, PubMed, Embase, Web of Science, PsycINFO, CINAHL and ProQuest, were searched for articles published from inception until February 
2021 without language or time restrictions. An additional search of OpenGrey was conducted for grey literature. A combination of Medical Subject Heading, Emtree terms and free terms [e.g., (postnatal OR postpartum OR delivery OR childbirth OR birth OR parturition OR labour OR pregnancy) AND (fatigue OR mental fatigue OR lassitude OR exhaust*) AND (randomized controlled trial OR controlled clinical trial OR cohort OR clinical trial) was used. The final search strategies applied are shown in Additional file 2. Moreover, a manual search of reference lists was performed to thoroughly identify relevant studies that were missed. Two authors (XXX and XXX) performed the search process independently.

\section{Eligibility/exclusion criteria for selecting studies}

The inclusion criteria of this review were as follows: (1) Study design: Clinical trials adopting randomized controlled trials (RCTs), quasi-experimental, beforeand-after or prospective cohort study designs; (2) Participants: women aged 18 years or over who had a healthy pregnancy; and (3) Intervention:

nonpharmacological interventions conducted during the women's postpartum period with the primary or secondary aim of decreasing fatigue symptoms. The intervention setting, frequency, timing and duration were not limited; (4) Comparison: usual care, placebo, waitlist or no interventions; and (5) Outcomes: the primary efficacy outcome was postpartum fatigue estimated as the rate or mean severity of fatigue. The second efficacy outcome was psychological variables, such as depression, anxiety or stress. The exclusion criteria were as follows: (1) duplicated publications (only the one with the most participants was included); (2) analyses of interventions based on postpartum fatigue in some special conditions, such as postpartum haemorrhage; and (3) studies without sufficient data to be extracted.

After removing duplicates, two authors $(X X X$ and $X X X)$ independently screened the studies according to the inclusion criteria in 2 steps: 1$)$ title and abstract screening and 2) full-text screening. A third author (XX) was consulted to reach a consensus when there was any uncertainty about the inclusion of an article.

\section{Data extraction}

A standardized data extraction sheet was used to extract important information from the included studies. The extracted data included first author, publication year, country, study design, population, sample size (trial/control), intervention details (e.g., type, frequency and duration), control, evaluation time points, assessment tools and outcomes. Two authors (XXX and XXX) independently extracted the data, and any inconsistencies were resolved by a third author (XX).

\section{Risk of bias summary}

For RCTs, the Cochrane risk-of-bias tool was used for the quality assessment[15]. Studies were assessed based on seven criteria (random sequence generation, allocation concealment, blinding of participants and researchers, blinding of outcome assessor, incomplete outcomes data, selective reporting and other bias). Bias was evaluated as a judgement (high or low or unclear), and then each included study was rated as having a high, moderate or low risk of bias. The Risk of Bias in Nonrandomized Studies of Interventions was used to assess the risk of bias of non-RCTs[16]. Seven domains are evaluated in this tool (confounding, selection of participants into the study, classification of intervention, deviation from the intended interventions, missing data, measurements of outcomes and selection of the reported results). Two authors (XXX and XXX) assessed the risk of bias and evidence quality separately. Disagreements were discussed with a third researcher $(X X)$ to reach a consistent conclusion.

\section{Data analysis}

We performed a meta-analysis utilizing Review Manager 5.3. For continuous outcomes, we calculated mean differences (MDs) and 95\% confidence intervals (Cls) if the outcomes were measured using the same tool. We used standardized mean differences (SMDs) and $95 \%$ Cls to combine studies when the same outcome was measured by adopting different tools[17]. For the SMD, $\leq 0.20,=0.50$, and $\geq 0.80$ are designated as small, moderate and large effect sizes, respectively[18]. The heterogeneity among the analysed trials was examined by standard chi-square and I-square statistics. If the $\mathrm{P}$ value was $>0.1$ or $\mathrm{I}^{2}<50 \%$, it indicated that there was no observed heterogeneity, and the researchers employed a fixed-effects model to combine the study results. If not, a randomeffects model analysis was used[19].

\section{Results}

The identified citations were imported into EndNote software and screened for duplicates. The initial search of 7 databases revealed 2955 references. A search of reference lists and OpenGrey revealed 3 other relevant studies. After removing 879 duplicates, the titles and abstracts of the remaining 2079 articles were screened, which excluded 2042 articles and left 37 full-text articles that were reviewed for eligibility. Twenty studies were excluded for being abstracts and protocols $(n=2)$, interventions were not conducted in the postpartum period $(n=6)$, could not find the full text $(n=1)$, studies published in another language ( $=2)$, studies without fatigue outcomes $(n=8)$ and studies not involving clinical interventions $(n=1)$. Ultimately, 17 studies were included in this systematic review, and 13 studies met the criteria for meta-analysis. A PRISMA flow diagram illustrating the detailed study selection process is shown in Fig. 1.

\section{Study characteristics}

The study characteristics are summarized in Table 1 . Studies were published between $2003 \sim 2020$. The 17 included articles were conducted in different countries: China $(n=5)$, Iran $(n=4)$, the USA $(n=2)$, Canada $(n=2)$, Turkey $(n=2)$, Australia $(n=1)$ and the UK $(n=1)$. A total of 2142 participants were included in this systematic review. The sample size ranged from 27 to 356 participants in each trial. Eleven of the 17 included studies employed a randomized controlled trial design.

Four types of interventions were undertaken: exercise [12, 20-23] $(n=5)$, psychoeducational intervention[13, 24-29] $(n=7)$, physical therapy[10, 11, 30] $(n=3)$ and drinking tea $[31,32](n=2)$. The intervention duration ranged from 1 day to 3 months. All included studies described the baseline assessments, with the scores for the intervention and control groups comparable at baseline. Additionally, the studies reported assessment scores immediately after the intervention and follow-up assessment scores at 1 month postintervention[31, 32], 6 weeks[24, 25, 28] and 9 weeks postintervention[24], 2 months postintervention[20], 3 months postintervention[21, 23, 25, 28] and 6 months[23] postintervention. These trials adopted the MFI-20, MFSC, PFS, VAS-F, FAS and FSC to evaluate the 
level of postpartum fatigue; the EDPS, DASS-21, CES-D, VAS for distress; and the PANAS and PSS were used to assess psychological variables such as depression, anxiety and stress.

\section{Risk of bias of the evidence}

The quality of the study designs was low to moderate overall. Several methodological limitations were observed in the critical appraisal. Quality assessments of the 11 included studies using an RCT design, with a risk of bias graph and risk of bias summary, are described in Fig. 2 and Fig. 3. All of the studies reported using randomization; however, two articles did not provide detailed information about the randomization method. Six studies reported sufficient details about allocation concealment. Blinding of the participants and researchers who delivered the interventions was not feasible because the interventions were easy to identify. Therefore, all of the studies are at a high risk of performance bias. Regarding detection bias, only four studies provided sufficient explanations. Except for one study, all RCT studies gave clear information about the incomplete outcome data. No reporting bias was found in the included RCTs.

The results of the quality appraisal of the six nonrandomized studies are displayed in Table 2. Bias due to confounding factors, selection of participants into the study and classification of interventions were low for all included nonrandomized studies. Three articles were at moderate risk of bias due to deviations from the intended intervention. All of the studies were reasonably reported and addressed missing data. In regard to bias in the measurement of outcomes, considering that the interventions were not blinded to the participants, all studies were judged as at moderate risk of bias. Bias in selection of the reported results was not observed. 
Table 1

Study characteristics $(n=17)$

\begin{tabular}{|c|c|c|c|c|c|c|c|c|c|}
\hline $\begin{array}{l}\text { First author } \\
\text { (publication } \\
\text { year) }\end{array}$ & Country & $\begin{array}{l}\text { Study } \\
\text { design }\end{array}$ & Population & $\begin{array}{l}\text { Sample size } \\
\text { (intervention/control) }\end{array}$ & $\begin{array}{l}\text { Intervention } \\
\text { trial }\end{array}$ & Frequency & Duration & Control & $\begin{array}{l}\text { Evalu } \\
\text { point }\end{array}$ \\
\hline \multicolumn{10}{|l|}{ Exercise $(n=5)$} \\
\hline $\begin{array}{l}\text { Ashrafinia[20] } \\
\text { (2015) }\end{array}$ & Iran & RCT & $\begin{array}{l}\text { Primiparous } \\
\text { women, } \\
\text { EPDS10 }\end{array}$ & $80(40 / 40)$ & $\begin{array}{l}\text { Pilates home } \\
\text { exercises }\end{array}$ & $\begin{array}{l}5 \text { times a } \\
\text { week (30 } \\
\text { min/session) }\end{array}$ & 8 weeks & $\begin{array}{l}\text { Routine } \\
\text { postpartum } \\
\text { care }\end{array}$ & $\begin{array}{l}\text { Basel } \\
\text { week: } \\
\text { postp }\end{array}$ \\
\hline $\begin{array}{l}\text { Dritsa[21] } \\
\text { (2008) }\end{array}$ & Canada & RCT & $\begin{array}{l}\text { Women in } \\
\text { the } \\
\text { postpartum } \\
\text { ( } 4-38 \\
\text { weeks), } \\
\text { EPDS } \geqq 10\end{array}$ & $88(46 / 42)$ & $\begin{array}{l}\text { Aerobic } \\
\text { exercise, } \\
\text { stretching and } \\
\text { strength } \\
\text { exercises }\end{array}$ & $\begin{array}{l}60-120 \\
\mathrm{~min} / \text { week, } 4 \\
\text { times }\end{array}$ & $\begin{array}{l}12 \\
\text { weeks }\end{array}$ & $\begin{array}{l}\text { Through } \\
\text { questionnaire } \\
\text { assessing } \\
\text { exercise } \\
\text { participation } \\
\text { once a } \\
\text { month }\end{array}$ & $\begin{array}{l}\text { Basel } \\
\text { treatr } \\
\text { mont } \\
\text { postt। }\end{array}$ \\
\hline $\begin{array}{l}\text { Ko[22] } \\
(2008)\end{array}$ & China & $\begin{array}{l}\text { A control } \\
\text { group } \\
\text { pre-/post- } \\
\text { program } \\
\text { design }\end{array}$ & $\begin{array}{l}\text { Postpartum } \\
\text { women who } \\
\text { were } \\
\text { "doing-the- } \\
\text { month" }\end{array}$ & $61(31 / 30)$ & $\begin{array}{l}\text { A low-intensity } \\
\text { exercise } \\
\text { program }\end{array}$ & 6 sessions & 3 weeks & Usual care & $\begin{array}{l}\text { Befor } \\
\text { the pr }\end{array}$ \\
\hline $\begin{array}{l}\text { Lee[23] } \\
(2016)\end{array}$ & UK & RCT & $\begin{array}{l}\text { Postnatal } \\
\text { women who } \\
\text { had given } \\
\text { birth } \\
\text { between } 6 \\
\text { weeks and } \\
\text { one year } \\
\text { previously }\end{array}$ & $65(33 / 32)$ & $\begin{array}{l}\text { Physical } \\
\text { activity } \\
\text { consultations } \\
\text { and a } \\
\text { moderate- } \\
\text { intensity } \\
\text { walking } \\
\text { programme }\end{array}$ & $\begin{array}{l}30-55 \\
\mathrm{~min} / \text { time, } \\
\text { one session } \\
\text { each week }\end{array}$ & $\begin{array}{l}10 \\
\text { weeks }\end{array}$ & $\begin{array}{l}\text { Received a } \\
\text { leaflet with } \\
\text { information } \\
\text { on physical } \\
\text { activity }\end{array}$ & $\begin{array}{l}\text { Basel } \\
\text { mont } \\
\text { mont } \\
\text { up. }\end{array}$ \\
\hline $\begin{array}{l}\text { Yang[12] } \\
\text { (2019) }\end{array}$ & China & RCT & $\begin{array}{l}\text { Postnatal } \\
\text { women who } \\
\text { had vaginal } \\
\text { delivery, } \\
\text { EPDS } \leqq 10\end{array}$ & $140(70 / 70)$ & $\begin{array}{l}\text { Moderate- } \\
\text { intensity } \\
\text { aerobic } \\
\text { exercise }\end{array}$ & $\begin{array}{l}\text { At least } 3 \\
\text { times a week } \\
(15 \text { min per } \\
\text { section })\end{array}$ & $\begin{array}{l}3 \\
\text { months }\end{array}$ & $\begin{array}{l}\text { Usual } \\
\text { postpartum } \\
\text { care }\end{array}$ & $\begin{array}{l}\text { Basel } \\
\text { week: } \\
\text { week: } \\
\text { postir }\end{array}$ \\
\hline \multicolumn{10}{|c|}{ Psychoeducational intervention $(n=7)$} \\
\hline $\begin{array}{l}\text { Doering[24] } \\
(2018)\end{array}$ & USA & $\begin{array}{l}\text { Quasi- } \\
\text { experimental }\end{array}$ & $\begin{array}{l}\text { Postpartum } \\
\text { women who } \\
\text { had a } \\
\text { healthy } \\
\text { singleton } \\
\text { newborn }\end{array}$ & $27(15 / 12)$ & $\begin{array}{l}\text { Helping U Get } \\
\text { Sleep }\end{array}$ & $\begin{array}{l}\text { A home visit } \\
\text { and } 4 \text { phone } \\
\text { calls }\end{array}$ & 4 weeks & $\begin{array}{l}\text { Health } \\
\text { education } \\
\text { related to } \\
\text { sleep for } \\
\text { attention } \\
\text { control }\end{array}$ & $\begin{array}{l}\text { Basel } \\
\text { postp } \\
\text { week: } \\
9\end{array}$ \\
\hline $\begin{array}{l}\text { Gholami[26] } \\
\text { (2017) }\end{array}$ & Iran & $\begin{array}{l}\text { A control } \\
\text { group } \\
\text { pre-/post- } \\
\text { program } \\
\text { design }\end{array}$ & $\begin{array}{l}\text { Women } \\
\text { who had } \\
\text { given birth }\end{array}$ & $120(40 / 40 / 40)$ & $\begin{array}{l}\text { Educational } \\
\text { behavioural } \\
\text { interventions } \\
\text { (face-to-face or } \\
\text { e-learning) }\end{array}$ & 3 times & 50 days & $\begin{array}{l}\text { Usual } \\
\text { postpartum } \\
\text { care }\end{array}$ & $\begin{array}{l}\text { Befor } \\
\text { the in }\end{array}$ \\
\hline $\begin{array}{l}\text { Giallo[25] } \\
(2014)\end{array}$ & Australia & RCT & $\begin{array}{l}\text { Mothers } \\
\text { who had a } \\
\text { child } \\
\text { younger } \\
\text { than } \\
\text { the age of } 6 \\
\text { months }\end{array}$ & $202(63 / 67 / 72)$ & $\begin{array}{l}\text { Intervention } \\
\text { group 1: } \\
\text { professionally } \\
\text { led support; } \\
\text { Intervention } \\
\text { group 2: self- } \\
\text { directed } \\
\text { written }\end{array}$ & $\begin{array}{l}\text { A workbook, } \\
\text { home visit, } \\
\text { and three } \\
\text { telephone } \\
\text { support calls; } \\
\text { reading the } \\
\text { workbook }\end{array}$ & 4 weeks & Usual care & $\begin{array}{l}\text { Basel } \\
12 \text { we } \\
\text { the bi }\end{array}$ \\
\hline $\begin{array}{l}\text { Milani[27] } \\
(2017)\end{array}$ & Iran & Clinical trial & $\begin{array}{l}\text { Healthy } \\
\text { postpartum } \\
\text { mothers, } \\
\text { EDPS }<10\end{array}$ & $276(92 / 184)$ & $\begin{array}{l}\text { A } \\
\text { comprehensive } \\
\text { postpartum } \\
\text { home care } \\
\text { program }\end{array}$ & $\begin{array}{l}2 \text { home visits } \\
\text { after } \\
\text { childbirth }\end{array}$ & 60 days & Usual care & $\begin{array}{l}\text { Prete: } \\
\text { postt! }\end{array}$ \\
\hline $\begin{array}{l}\text { Ozcan[13] } \\
(2020)\end{array}$ & Turkey & RCT & $\begin{array}{l}\text { Primiparous } \\
\text { women }\end{array}$ & $117(58 / 59)$ & $\begin{array}{l}\text { Levin's } \\
\text { conservation } \\
\text { model }\end{array}$ & $\begin{array}{l}8 \text { sessions, } \\
\text { each session } \\
\text { lasted 60- } \\
120 \text { min. }\end{array}$ & $\begin{array}{l}12 \\
\text { weeks }\end{array}$ & $\begin{array}{l}\text { Standard } \\
\text { nursing care }\end{array}$ & $\begin{array}{l}\text { Prete: } \\
\text { postt! }\end{array}$ \\
\hline
\end{tabular}

Notes: RCT: randomized clinical trial; MFI-20: Multidimensional Fatigue Inventory; PFS: Postpartum Fatigue Scale; VAS-F: visual analogue scale for fatigue; F. Modified Fatigue Symptoms Checklist; FSC: Fatigue Symptom Checklist; EDPS: Edinburgh Postnatal Depression Scale; DASS-21: Depression, Anxiety and Str of the Center for Epidemiologic Studies Depression Scale; PANAS: Positive and Negative Affect Schedule; PSS: Perceived Stress Scale; AGWBI: Adapted Gene 


\begin{tabular}{|c|c|c|c|c|c|c|c|c|c|}
\hline $\begin{array}{l}\text { First author } \\
\text { (publication } \\
\text { year) }\end{array}$ & Country & $\begin{array}{l}\text { Study } \\
\text { design }\end{array}$ & Population & $\begin{array}{l}\text { Sample size } \\
\text { (intervention/control) }\end{array}$ & $\begin{array}{l}\text { Intervention } \\
\text { trial }\end{array}$ & Frequency & Duration & Control & $\begin{array}{l}\text { Evalu } \\
\text { point }\end{array}$ \\
\hline Stremler[28] & Canada & RCT & $\begin{array}{l}\text { Primiparous } \\
\text { women }\end{array}$ & $246(123 / 123)$ & $\begin{array}{l}\text { Behavioural- } \\
\text { educational } \\
\text { sleep } \\
\text { intervention }\end{array}$ & $\begin{array}{l}\text { A } 45-60 \\
\text { minute } \\
\text { meeting, a } \\
20 \text { page } \\
\text { booklet, and } \\
3 \text { phone } \\
\text { contacts. }\end{array}$ & 4 weeks & Usual care & $\begin{array}{l}\text { Basel } \\
12 \mathrm{w \epsilon}\end{array}$ \\
\hline
\end{tabular}

(2013)

\begin{tabular}{|c|c|c|c|c|c|c|c|c|c|}
\hline $\begin{array}{l}\text { Troy[29] } \\
(2003)\end{array}$ & USA & $\begin{array}{l}\text { Quasi- } \\
\text { experimental }\end{array}$ & $\begin{array}{l}\text { Healthy } \\
\text { primiparous } \\
\text { postpartum } \\
\text { mothers }\end{array}$ & $68(32 / 36)$ & $\begin{array}{l}\text { The Tiredness } \\
\text { Management } \\
\text { Guide as a } \\
\text { self-care } \\
\text { intervention }\end{array}$ & $\begin{array}{l}\text { Women in } \\
\text { the } \\
\text { intervention } \\
\text { group were } \\
\text { asked to use } \\
\text { the TMG } \\
\text { whenever } \\
\text { they felt tired }\end{array}$ & 5 weeks & $\begin{array}{l}\text { Usual } \\
\text { postpartum } \\
\text { care }\end{array}$ & $\begin{array}{l}\text { Fatigı } \\
\text { asses } \\
\text { times } \\
\text { befor } \\
\text { bed a } \\
\text { on ris } \\
\text { Tuesc } \\
\text { eveni } \\
\text { Frida! }\end{array}$ \\
\hline
\end{tabular}

\section{Physical therapy $(n=3)$}

\begin{tabular}{|c|c|c|c|c|c|c|c|c|c|}
\hline $\begin{array}{l}\text { Funda[10] } \\
(2020)\end{array}$ & Turkey & RCT & $\begin{array}{l}\text { Primiparous } \\
\text { and had a } \\
\text { vaginal } \\
\text { delivery at } \\
\text { the } 37 \text { th to } \\
40 \text { th weeks } \\
\text { of gestation }\end{array}$ & $80(40 / 40)$ & $\begin{array}{l}\text { Mother-infant } \\
\text { skin-to-skin } \\
\text { contact }\end{array}$ & 1 time & $\begin{array}{l}30 \\
\text { minutes }\end{array}$ & $\begin{array}{l}\text { Usual } \\
\text { postpartum } \\
\text { care }\end{array}$ & $\begin{array}{l}\text { Befor } \\
\text { the in }\end{array}$ \\
\hline $\begin{array}{l}\text { Hsieh[30] } \\
\text { (2017) }\end{array}$ & China & $\begin{array}{l}\text { Quasi- } \\
\text { experimental }\end{array}$ & $\begin{array}{l}\text { Healthy } \\
\text { postpartum } \\
\text { women }\end{array}$ & $356(94 / 264)$ & $\begin{array}{l}\text { Take warm } \\
\text { showers }\end{array}$ & 1 time & $\begin{array}{l}20 \\
\text { minutes }\end{array}$ & $\begin{array}{l}\text { Routine } \\
\text { postpartum } \\
\text { care }\end{array}$ & $\begin{array}{l}\text { The fi } \\
\text { postp } \\
\text { and tl } \\
\text { postp }\end{array}$ \\
\hline $\begin{array}{l}\text { Vaziri[11] } \\
\text { (2017) }\end{array}$ & Iran & RCT & $\begin{array}{l}\text { Primiparous } \\
\text { women with } \\
\text { normal } \\
\text { vaginal } \\
\text { delivery }\end{array}$ & $56(29 / 27)$ & $\begin{array}{l}\text { Breathe } \\
\text { lavender oil } \\
\text { aroma }\end{array}$ & $\begin{array}{l}3 \text { times, } 10- \\
15 \text { minutes } \\
\text { each time }\end{array}$ & 1 day & $\begin{array}{l}\text { Sesame oil } \\
\text { used as } \\
\text { placebo }\end{array}$ & $\begin{array}{l}\text { Basel } \\
\text { the fir } \\
\text { interv } \\
\text { the to } \\
\text { morn } \\
\text { asses }\end{array}$ \\
\hline \multicolumn{10}{|c|}{ Drinking tea $(n=2)$} \\
\hline $\begin{array}{l}\text { Chang[32] } \\
(2015)\end{array}$ & China & RCT & $\begin{array}{l}\text { Postnatal } \\
\text { women in } \\
\text { their sixth } \\
\text { postpartum } \\
\text { week with } \\
\text { poor sleep } \\
\text { quality } \\
\text { (PSQS } \\
\text { score } \geqq 16)\end{array}$ & $80(40 / 40)$ & $\begin{array}{l}\text { Drink one cup } \\
\text { of chamomile } \\
\text { tea }\end{array}$ & Every day & 2 weeks & $\begin{array}{l}\text { No } \\
\text { intervention, } \\
\text { regular } \\
\text { postpartum } \\
\text { care }\end{array}$ & $\begin{array}{l}\text { Basel } \\
2 \text { and } \\
\text { post } \\
\text { interv }\end{array}$ \\
\hline Chen[31] & China & RCT & $\begin{array}{l}\text { Postnatal } \\
\text { women with } \\
\text { poor sleep } \\
\text { quality } \\
\text { (PSQS } \\
\text { score } \geqq 16 \text { ) }\end{array}$ & $80(40 / 40)$ & $\begin{array}{l}\text { Drink one cup } \\
\text { of Lavender } \\
\text { tea }\end{array}$ & Every day & 2 weeks & $\begin{array}{l}\text { Regular } \\
\text { postpartum } \\
\text { care }\end{array}$ & $\begin{array}{l}\text { Basel } \\
\text { week } \\
\text { and } 4 \\
\text { postt }\end{array}$ \\
\hline
\end{tabular}

(2015)

Notes: RCT: randomized clinical trial; MFI-20: Multidimensional Fatigue Inventory; PFS: Postpartum Fatigue Scale; VAS-F: visual analogue scale for fatigue; F. Modified Fatigue Symptoms Checklist; FSC: Fatigue Symptom Checklist; EDPS: Edinburgh Postnatal Depression Scale; DASS-21: Depression, Anxiety and Str of the Center for Epidemiologic Studies Depression Scale; PANAS: Positive and Negative Affect Schedule; PSS: Perceived Stress Scale; AGWBI: Adapted Gen€ 
Table 2

Results of the quality appraisal of nonrandomized studies $(n=6)$

\begin{tabular}{|c|c|c|c|c|c|c|c|c|}
\hline & Domain 1 & Domain 2 & Domain 3 & Domain 4 & Domain 5 & Domain 6 & Domain 7 & Judgement \\
\hline $\begin{array}{l}\text { Doering } \\
(2018)\end{array}$ & Low & Low & Low & Low & Low & Moderate & Low & Moderate \\
\hline $\begin{array}{l}\text { Gholami } \\
(2017)\end{array}$ & Low & Low & Low & Low & Low & Moderate & Low & Moderate \\
\hline $\begin{array}{l}\text { Hsieh } \\
(2017)\end{array}$ & Low & Low & Low & Low & Low & Moderate & Low & Moderate \\
\hline $\begin{array}{l}\text { Ko } \\
\text { (2008) }\end{array}$ & Low & Low & Low & Moderate & Low & Moderate & Low & Moderate \\
\hline $\begin{array}{l}\text { Milani } \\
(2017)\end{array}$ & Low & Low & Low & Moderate & Low & Moderate & Low & Moderate \\
\hline $\begin{array}{l}\text { Troy } \\
\text { (2003) }\end{array}$ & Low & Low & Low & Moderate & Low & Moderate & Low & Moderate \\
\hline
\end{tabular}

\section{Meta-analysis results}

For the purposes of meta-analysis, postpartum interventions were grouped into four broad categories: exercise, psychoeducational interventions, physical therapy and drinking tea. Only one trial[27] reported the postpartum fatigue rate. Three trials[21, 23, 24] without sufficient original data for meta-analysis were thus not included. Ultimately, thirteen studies that reported the mean fatigue scores and standard deviations were included in the meta-analysis. For the secondary outcomes, since more than two studies reported depression, a statistical combination was performed regarding this psychological outcome.

\section{Effectiveness of exercise}

Figure 4 shows the effect sizes of the exercise intervention in terms of fatigue and depression. For the primary outcome, three trials showed a postassessment of fatigue. The heterogeneity was $\mathrm{I}^{2}=95 \%(\mathrm{P}<0.0001)$, so a random-effects model was applied. The pooled SMD was $-1.74(95 \% \mathrm{Cl}=-2.61$ to $-0.88, Z=3.94$, $\mathrm{P}<0.0001$ ), demonstrating that the exercise intervention had a better effect on decreasing fatigue symptoms. For the secondary outcome, depression data were presented in two trials. No significant effects on reducing depression were indicated. Significant heterogeneity was not detected $\left(I^{2}=46 \%\right.$, $\left.P=0.17\right)$, so a fixed-effect model was used. The pooled SMD was $-0.05(95 \% \mathrm{Cl}=-0.33$ to $0.24, \mathrm{Z}=0.31, \mathrm{P}=0.75)$.

\section{Effectiveness of physical therapy}

Three trials showed the effectiveness of physical therapy on posttreatment fatigue severity (Fig. 6). Significant improvements were observed in the participants who received physical therapy in comparison with the participants in the control groups. There was substantial evidence of high heterogeneity $\left(\mathrm{I}^{2}\right.$ $=74 \%, \mathrm{P}=0.02)$, so a random-effect model was applied. The pooled SMD was $-0.50(95 \% \mathrm{Cl}=-0.96$ to $-0.03, \mathrm{Z}=2.09, \mathrm{P}=0.04)$.

\section{Effectiveness of drinking tea}

The effectiveness of drinking tea on fatigue and depression at postintervention and at the 2-week follow-up were presented in two trials (Fig. 7). At postintervention, significant differences were noted between the intervention and control groups in regard to fatigue and depression. No significant heterogeneity was found in terms of fatigue and depression $\left(I^{2}=0 \%, P=0.44 ; I^{2}=0 \%, P=0.74\right)$. Therefore, a fixed-effects model was used. The pooled $M D$ was $-3.12(95 \% \mathrm{Cl}=-5.44$ to $-0.80, \mathrm{Z}=2.64, \mathrm{P}=0.008)$ and $-2.89(95 \% \mathrm{Cl}=-4.30$ to $-1.49, \mathrm{Z}=4.04, \mathrm{P}<0.0001)$.

At the 2-week follow-up, the results suggested that there were no significant differences between the intervention and control groups in regard to fatigue. There was no evidence of significant heterogeneity $\left(\mathrm{I}^{2}=0 \%, \mathrm{P}=0.59\right)$. The pooled $\mathrm{MD}$ was $-1.38(95 \% \mathrm{Cl}=-3.58$ to $0.81, \mathrm{Z}=1.24, \mathrm{P}=0.22)$. The results showed that tea consumption had significant effects on depression at the 2-week follow-up. We used a fixed-effects model because the heterogeneity was $\left.\right|^{2}=0 \%(P=$ $0.77)$. The pooled MD was $-2.05(95 \% \mathrm{Cl}=-3.47$ to $-0.62, \mathrm{Z}=2.82, \mathrm{P}=0.005)$.

\section{Discussion}

\section{Main findings}

This systematic review identified a total of seventeen published clinical trials examining nonpharmacological interventions (including exercise, psychoeducational interventions, physical therapy and drinking tea) on postpartum fatigue in postnatal women. Thirteen studies involving 1686 participants were included in the meta-analysis to examine the respective effectiveness of each type of intervention. 
The findings of the meta-analysis suggest that exercise intervention significantly reduces symptoms of postpartum fatigue $(\mathrm{SMD}=-1.74,95 \% \mathrm{Cl}=-2.61$ to $-0.88, Z=3.94, P<0.0001)$. This is consistent with the findings of previous studies[33,34], which clarified the necessities and benefits of physical exercise for the overall health of postpartum women. A meta-analysis[35] indicated that postpartum exercise was beneficial for decreasing postpartum fatigue (SMD = 0.36). The benefits of exercise on fatigue are worth affirming. However, postpartum women are still at high risk for physical inactivity and rarely understand how to engage in postpartum exercise[33]. The American College of Obstetricians and Gynaecologists (ACOG) suggested that physical activity, including performing muscle-toning exercises, could be restarted 6 weeks after childbirth if the delivery was uncomplicated[36]. Daily $20 \sim 30$ min of regular moderateto-intense exercise is recommended[37]. A previous study reported that supervised postpartum exercise lasting more than eight weeks is suggested for reducing postpartum fatigue[14]. Therefore, clinical staff should give sufficient explanations and evidence-based instructions for postnatal exercises to help reduce women's postpartum fatigue and facilitate their postpartum rehabilitation.

For depression, the results showed that there were no significant differences between the two groups $(\mathrm{SMD}=-0.05,95 \% \mathrm{Cl}=-0.33$ to $0.24, \mathrm{Z}=0.31, \mathrm{P}=0.75)$. This is inconsistent with the findings of a previous meta-analysis[38], which reported that exercise reduced symptoms of depression (SMD $=-0.81,95 \% \mathrm{Cl}=-1.53$ to -0.10 ) of mothers who had been diagnosed with depression. The insignificant results may be explained by the fact that the two studies[12, 22] in our review did not include participants with obvious depressed symptoms at baseline. Therefore, a significant reduction in depression was difficult to observe after the exercise intervention. It is worth noting that in Yang et al.[12]'s study, women reported feelings of mood relaxation and pressure relief after the intervention, which indicated that exercise may have potential efficacy for women's mental health. More research conducting exercise interventions among puerperae who have severe symptoms of psychological problems is needed to confirm the true effect of exercise on mothers' psychological well-being.

Five studies performing psychoeducational interventions showed no obvious differences in postpartum fatigue and depression between the two groups at either the postintervention or 8-week follow-up. Although psychoeducational interventions usually involve various aspects associated with postnatal health, including fatigue, sleep, infection, nutrition, breastfeeding and so on, this type of intervention was mostly delivered via home visits, leaflet/booklets and home calls. It is difficult to guarantee the good compliance of participants due to a lack of sufficient supervision and guidance. Therefore, it may be difficult to perform psychoeducational interventions effectively. With the development of rapid electronic technology, people's requests for health services have also facilitated instant communication and promoted efficiency in the transmission of information[39]. A previous study reported that web-based interventions had better effects on improving postnatal depression than home-based postnatal psychoeducational interventions[40] and suggested that web-based interventions should be introduced to mothers for better postnatal care. Hence, psychoeducational interventions could be combined with internet technology[41] or smartphones[42] in the future to improve the participation of women, observe the compliance of the participants and better manage the intervention implementation.

The findings from our meta-analysis of three studies $[10,11,30]$ suggested that physical therapy was an effective treatment in relieving postpartum fatigue $(\mathrm{SMD}=-0.50,95 \% \mathrm{Cl}=-0.96$ to $-0.03, \mathrm{Z}=2.09, \mathrm{P}=0.04)$. The intervention frequency of the included physical therapies ranged from $1 \sim 3$ sessions. Each session lasted $10 \sim 30$ minutes. These interventions were completed in 1 day. Physical therapies have the advantages of a short intervention time and good controllability. Warm showers, as a comfort measure, are closely associated with increased relaxation and tension reduction[43]. It is a safe and effective measure for healthy, labouring women who are experiencing physical and psychological issues[43]. The benefits of lavender oil on postpartum fatigue were reported in another RCT conducted among pregnant women[44]. Participants in either the lavender and footbath or lavender alone group showed that fatigue was improved significantly at 6 weeks postpartum. Although the efficacy of mother-infant skin-to-skin on fatigue was not observed in Funda et al.'s study, a recently published meta-analysis demonstrated that mother-infant skin-to-skin was a cost-effective, simple and feasible approach for postpartum depression[45]. Considering that physical therapies are relatively safe and effective, physical therapies such as footbaths[44], reflexology[46], warm showers[30] and lavender oil[11], which show effects on fatigue reduction and mental health improvement, could be used in combination to enhance the intervention efficacy. Among the three included studies, only Vaziri et al.'s study examined the efficacy of lavender oil aroma for psychological outcomes, including distress and mood, and positive effects were observed. More research is needed to explore its effectiveness for mental health.

In terms of drinking tea, the results showed that there were significant differences in postpartum fatigue between the two groups at postintervention (MD= $-3.12,95 \% \mathrm{Cl}=-5.44$ to $-0.80, \mathrm{Z}=2.64, \mathrm{P}=0.008$ ), but no significant differences were found at the 2-week follow-up. The positive effects of drinking tea on relieving depression were obvious at the postintervention and 2-week follow-up. Postnatal women were required to smell (appreciate) the aroma before drinking the tea. Aromatherapy has been used for pain and anxiety relief, relaxation, and creating a pleasant feeling in mothers[47-49], which could help to relieve fatigue and depressive emotions. Women in the intervention group reported the benefits of drinking chamomile tea to be facilitating emotional stability and relaxation and having an aromatic fragrance, which could calm restlessness, facilitate the postnatal paternity relationship, and alleviate postpartum fatigue[50]. However, the positive effects did not last long after the intervention. Thus, multiple daily consumption of tea may be recommended to assess its lasting effect in consideration of its convenience.

\section{Strengths and limitations}

To the best of our knowledge, this is the first systematic review and meta-analysis on nonpharmacological interventions for reducing postpartum fatigue. This review was rigorous and based on the Preferred Reporting Items for Systematic Reviews and Meta-Analysis statements as well as a prospective registered protocol. Another strength was that randomized controlled trials and controlled clinical trials were included in this review, which provides good standards for evidence-based research.

There were some limitations of this systematic review. First, a limitation of the review was that non-English electronic databases were not searched, which may cause language bias[51]. Second, the number of included studies for each type of intervention was small. The heterogeneity in the statistical combinations of exercise, psychoeducational intervention and physical therapy was significant. These factors may have an impact on the reliability of the pooled results. Third, a limitation lies in the risk of bias within the included studies. The potential biases may have influenced the reported effect estimates; therefore, caution is required when interpreting the findings of our study. 


\section{Conclusions}

The results from this systematic review and meta-analysis provide evidence that nonpharmacological interventions, including exercise, physical therapy and drinking tea, are effective in reducing postpartum fatigue. Detailed and evidence-based instructions involving exercise frequency and duration should be offered to puerperae. Considering that physical therapies are relatively safe, they could be used in combination to enhance the intervention efficacy. Multiple daily cups of tea may be recommended to assess its lasting effect. The effects of psychoeducation were not noted, and future research could integrate psychoeducation with internet technology or smartphones to improve the compliance of the participants. The effectiveness of fatigue-related nonpharmacological interventions on psychological outcomes still needs to be further investigated due to the limited number of studies.

\section{Abbreviations}

RCT: Randomized controlled trial; CCT: Controlled clinical trial; PROSPERO: International prospective register of systematic reviews; PRISMA: Preferred reporting items for systematic reviews and meta-analyses; MFI-20: Multidimensional Fatigue Inventory; PFS: Postpartum Fatigue Scale; VAS-F: visual analogue scale for fatigue; FAS: Fatigue Assessment Scale; MFSC: Modified Fatigue Symptoms Checklist; FSC: Fatigue Symptom Checklist; EDPS: Edinburgh Postnatal Depression Scale; DASS-21: Depression, Anxiety and Stress Scale-21; CES-D Scale: Chinese version of the Center for Epidemiologic Studies Depression Scale; PANAS: Positive and Negative Affect Schedule; PSS: Perceived Stress Scale; AGWBI: Adapted General Well-Being Index; Cl: Confidence interval; MD: Mean difference; SMD: standardized mean difference.

\section{Declarations}

\section{Ethics approval and consent to participate}

Not applicable.

\section{Consent for publication}

Not applicable.

\section{Acknowledgements}

Not applicable.

\section{Authors' contributions}

This systematic review was conceived and drafted by JLQ under the supervision of XYY; JLQ and SWS independently searched electronic databases and screened titles and abstracts. JLQ and SWS were responsible for reviewing the included studies. Disagreements were discussed with a third researcher (LL) to reach a consistent conclusion. All authors contributed to the draft revision.

\section{Funding}

This work was supported by Medical and Health Research Project of Zhejiang Province (grant number 2020KY173)

\section{Competing interests}

None declared.

\section{Availability of data and materials}

Data will be available from the corresponding author upon reasonable request

\section{References}

1. Badr HA, Zauszniewski JA: Meta-analysis of the predictive factors of postpartum fatigue. Applied Nursing Research Anr 2017, $36: 122$.

2. Senol DK, Yurdakul M, Ozkan SA: The effect of maternal fatigue on breastfeeding. NIGER J CLIN PRACT 2019, $22(12): 1662$.

3. Wilson N, Lee JJ, Bei B: Postpartum fatigue and depression: A systematic review and meta-analysis. J AFFECT DISORDERS 2018.

4. Henderson J, Alderdice F, Redshaw M: Factors associated with maternal postpartum fatigue: an observationalstudy. BMJ OPEN $2019,9(7):$ e25927.

5. Lai, Ya-Ling, Stocker, Joel, Chan, Te-Fu, Liu, Yi, Hung, Chich-Hsiu: Postpartum fatigue, baby-care activities, and maternal-infant attachment of vaginal and cesarean births following rooming-in. Applied nursing research: ANR 2015, 28(2):116-120.

6. Rychnovsky JD: Postpartum fatigue in the active-duty military woman. J Obstet Gynecol Neonatal Nurs 2007, 36(1):38-46.

7. Kurth E, Kennedy HP, Spichiger E, Hösli I, Stutz EZ: Crying babies, tired mothers: what do we know? A systematic review. MIDWIFERY 2011, 27(2):187-194.

8. Lai YL, Hung CH, Stocker J, Chan TF, Liu Y: Postpartum fatigue, baby-care activities, and maternal-infant attachment of vaginal and cesarean births following rooming-in. APPL NURS RES 2015, 28(2):116-120.

9. Cindy-Lee, Dennis, Simone, Vigod: Preventing postpartum depression: fatigue management is a place to start. Evidence Based Nursing 2019. 
10. Funda Tosun G, Salime M, Özgürlük İ: The effect of mother-infant skin-to-skin contact on the involution process and maternal postpartum fatigue during the early postpartum period. WOMEN HEALTH 2020, 60(6):707-718.

11. Vaziri F, Shiravani M, Najib F, Pourahmad S, Salehi A, Yazdanpanahi Z: Effect of lavender oil aroma in the early hours of postpartum period on maternal pains, fatigue, and mood: A randomized clinical trial. International Journal of Preventive Medicine 2017, 8(1).

12. Yang C, Chen C: Effectiveness of aerobic gymnastic exercise on stress, fatigue, and sleep quality during postpartum: A pilot randomized controlled trial. INT J NURS STUD 2018, 77:1-7.

13. Ozcan S, Eryilmaz G: Using Levine's conservation model in postpartum care: a randomized controlled trial. HEALTH CARE WOMEN IN $2020: 1-21$.

14. Liu N, Wang J, Chen DD, Sun WJ, Li P, Zhang W: Effects of exercise on pregnancy and postpartum fatigue: A systematic review and meta-analysis. Eur J Obstet Gynecol Reprod Biol 2020, 253:285-295.

15. Higgins JP, Altman DG, Gotzsche PC, Juni P, Moher D, Oxman AD, Savovic J, Schulz KF, Weeks L, Sterne JA: The Cochrane Collaboration's tool for assessing risk of bias in randomised trials. BMJ 2011, 343:d5928.

16. Sterne JA, Hernán MA, Reeves BC, Savović J, Berkman ND, Viswanathan M, Henry D, Altman DG, Ansari MT, Boutron I et al: ROBINS-I: a tool for assessing risk of bias in non-randomised studies of interventions. BMJ 2016, 355:i4919.

17. Wen J, Li Y: The Selection of a Summary Statistic for Use in Meta-analysis. CHINESE JOURNAL OF EVIDENCE-BASED MEDICINE 2007(08):606-613.

18. Cohen J: Statistical Power Analysis for the Behavioral Sciences; 1988.

19. Higgins JPT, Thompson SG: Quantifying heterogeneity in a meta-analysis. STAT MED 2002, 21(11):1539-1558.

20. Ashrafinia F, Mirmohammadali M, Rajabi H, Kazemnejad A, Haghighi KS, Amelvalizadeh M: Effect of Pilates exercises on postpartum maternal fatigue. SINGAP MED J 2015, 56(3):169-173.

21. Dritsa M, Da Costa D, Dupuis G, Lowensteyn I, Khalife S: Effects of a home-based exercise intervention on fatigue in postpartum depressed women: Results of a randomized controlled trial. ANN BEHAV MED 2008, 35(2):179-187.

22. Ko Y, Yang C, Chiang L: Effects of Postpartum Exercise Program on Fatigue and Depression During “Doing-the-Month" Period. The journal of nursing research 2008, 16:177-186.

23. Lee AS, McInnes RJ, Hughes AR, Guthrie W, Jepson R: The Effect of the More Active MuMs in Stirling Trial on Body Composition and Psychological WellBeing among Postnatal Women. Journal of pregnancy 2016, 2016:4183648.

24. Doering JJ, Dogan S: A Postpartum Sleep and Fatigue Intervention Feasibility Pilot Study. BEHAV SLEEP MED 2018, 16(2):185-201.

25. Giallo R, Cooklin A, Dunning M, Seymour M: The Efficacy of an Intervention for the Management of Postpartum Fatigue. JOGNN: Journal of Obstetric, Gynecologic \& Neonatal Nursing 2014, 43(5):598-613.

26. Gholami Z, Mohammadirizi S, Bahadoran P: Study of the Impact of Educational Behavioral Interventions on Fatigue in Mothers in the Postpartum Period in the Groups of Face-to-Face and Electronic Training. Iran J Nurs Midwifery Res 2017, 22(6):465-470.

27. Milani H, Amiri P, Mohseny M, Abadi A, Vaziri S, Vejdani M: Postpartum home care and its effects on mothers' health: A clinical trial. J RES MED SCI 2017, 22(1).

28. Stremler R, Hodnett E, Kenton L, Lee K, Weiss S, Weston J, Willan A: Effect of behavioural-educational intervention on sleep for primiparous women and their infants in early postpartum: multisite randomised controlled trial. BMJ 2013, 346:f1164.

29. Troy NW, Dalgas-Pelish P: The effectiveness of a self-care intervention for the management of postpartum fatigue. APPL NURS RES 2003, 16(1):38-45.

30. Hsieh CH, Chen CL, Chung FF, Lin SY: Efficacy of Warm Showers on Postpartum Fatigue Among Vaginal-Birth Taiwanese Women: a Quasi-Experimental Design. RES THEOR NURS PRACT 2017, 31(2):96-106.

31. Chen SL, Chen CH: Effects of Lavender Tea on Fatigue, Depression, and Maternal-Infant Attachment in Sleep-Disturbed Postnatal Women. Worldviews Evid Based Nurs 2015, 12(6):370-379.

32. Chang SM, Chen $\mathrm{CH}$ : Effects of an intervention with drinking chamomile tea on sleep quality and depression in sleep disturbed postnatal women: a randomized controlled trial. J ADV NURS 2016, 72(2):306-315.

33. Adeniyi AF, Ogwumike OO, Bamikefa TR: Postpartum Exercise among Nigerian Women: Issues Relating to Exercise Performance and Self-Efficacy. ISRN Obstet Gynecol 2013, 2013:294518.

34. Larson-Meyer DE: Effect of Postpartum Exercise on Mothers and their Offspring: A Review of the Literature. Obesity Research 2002, 10(8):841-853.

35. Liu N, Wang J, Chen DD, Sun WJ, Zhang W: Effects of exercise on pregnancy and postpartum fatigue: A systematic review and meta-analysis. EUR J OBSTET GYN R B 2020, 253.

36. Artal R, O'Toole M: Guidelines of the American College of Obstetricians and Gynecologists for exercise during pregnancy and the postpartum period. BRIT J SPORT MED 2003, 37(1):6-12.

37. ACOG: Committee Opinion No. 650 Summary: Physical Activity and Exercise During Pregnancy and the Postpartum Period. OBSTET GYNECOL 2015, 126(6):1326-1327.

38. Daley A, Jolly K, MacArthur C: The effectiveness of exercise in the management of post-natal depression: systematic review and meta-analysis. FAM PRACT 2009, 26(2):154-162.

39. Ashish A, Sameer K, Florence D, Sara F, Jason R, Thomas U, Jean-Frédéric C, Shirley M, Bruce S: Unmet Communication and Information Needs for Patients with IBD: Implications for Mobile Health Technology. British Journal of Medicine \& Medical Research 2016, 12(3):S48-S49.

40. Jiao N, Zhu L, Chong YS, Chan WS, Luo N, Wang W, Hu R, Chan YH, He HG: Web-based versus home-based postnatal psychoeducational interventions for first-time mothers: A randomised controlled trial. INT J NURS STUD 2019, 99:103385.

Page 10/14 
41. Bevan JR, Thapar A, Rice F, Beeching H, Cichosz R, Mars B, Smith DJ, Merry S, Stallard P, Jones I et al: A Web-Based Psychoeducational Intervention for Adolescent Depression: Design and Development of MoodHwb. JMIR Ment Health 2018, 5(1):e13.

42. Chan KL, Leung WC, Tiwari A, Or KL, Ip P: Using Smartphone-Based Psychoeducation to Reduce Postnatal Depression Among First-Time Mothers: Randomized Controlled Trial. JMIR MHEALTH UHEALTH 2019, 7(5):e12794.

43. Stark MA: Therapeutic showering in labor. CLIN NURS RES 2013, 22(3):359-374.

44. Fatemeh ED, Sakineh MAC, Mojgan M, Mohsen T, Reza B, Somayeh Z: Effect of Lavender cream with or without footbath on sleep quality and fatigue in pregnancy and postpartum: a randomized controlled trial. WOMEN HEALTH 2018:1.

45. Kirca N, Adibelli D: Effects of mother-infant skin-to-skin contact on postpartum depression: A systematic review. PERSPECT PSYCHIATR C 2021.

46. Son CM, Ja LE: Effects of Foot-Reflexology Massage on Fatigue, Stress and Postpartum Depression in Postpartum Women. J KOREAN ACAD NURS 2015, 45(4):587-594.

47. Yazdkhasti M, Pirak A: The effect of aromatherapy with lavender essence on severity of labor pain and duration of labor in primiparous women. Complement Ther Clin Pract 2016, 25:81-86.

48. Pollard K: Introducing aromatherapy as a form of pain management into a delivery suite. J Assoc Chart Physiotherapists Womens Health 2008(103):1216.

49. Cavanagh HMA, Wilkinson JM: Biological activities of Lavender essential oil. PHYTOTHER RES 2002.

50. Chang SM, Chen $\mathrm{CH}$ : Effects of an intervention with drinking chamomile tea on sleep quality and depression in sleep disturbed postnatal women: a randomized controlled trial. Journal of Advanced Nursing 2016, 72(2):306-315.

51. Morrison A, Polisena J, Husereau D, Moulton K, Clark M, Fiander M, Mierzwinski-Urban M, Clifford T, Hutton B, Rabb D: The effect of English-language restriction on systematic review-based meta-analyses: a systematic review of empirical studies. INT J TECHNOL ASSESS 2012, 28(2):138-144.

\section{Figures}

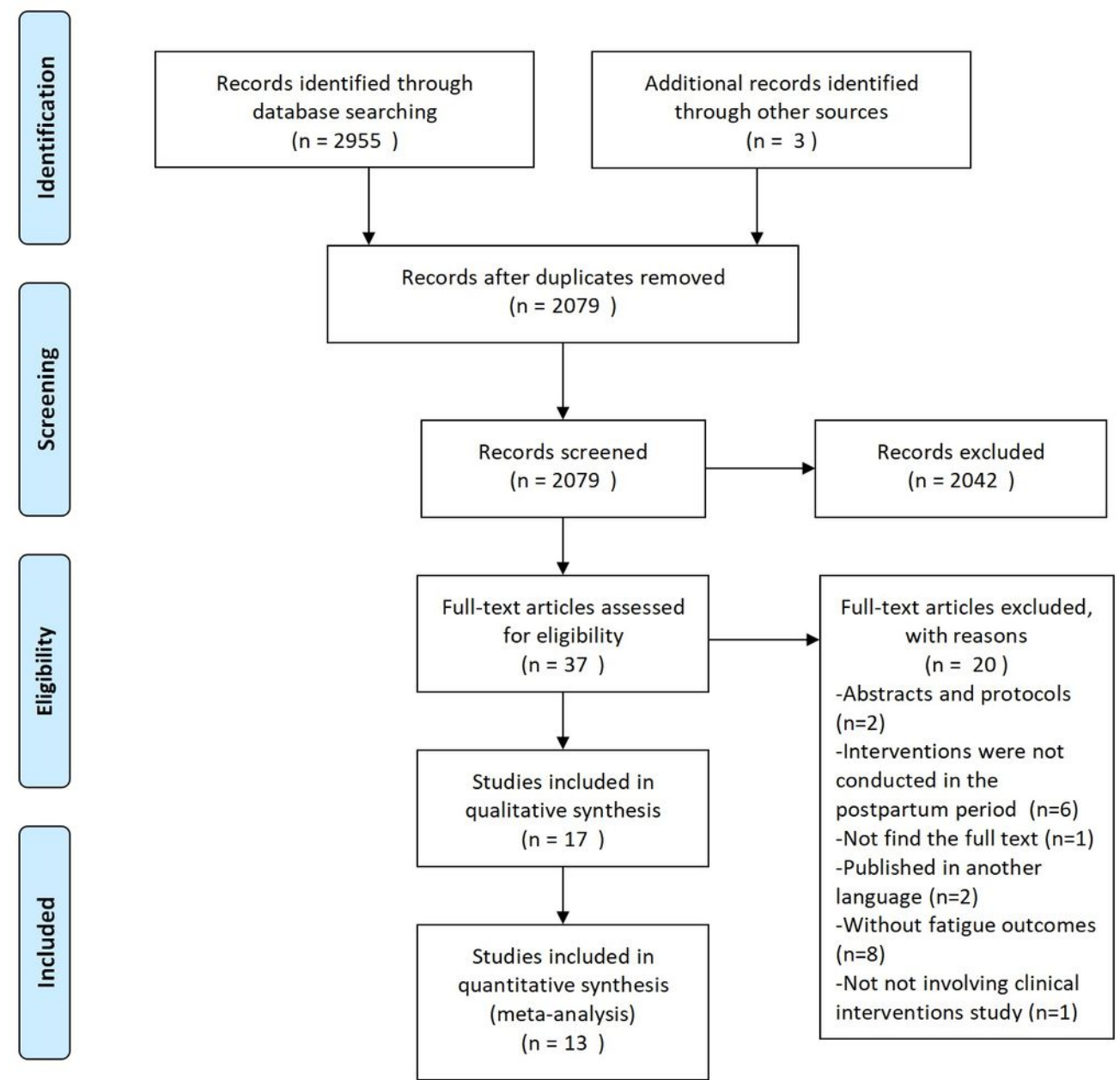

Figure 1

PRISMA flow diagram 


\begin{tabular}{|c|c|c|c|c|c|c|c|}
\hline & 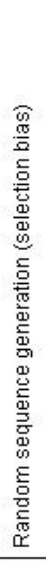 & 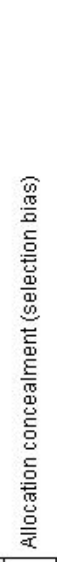 & 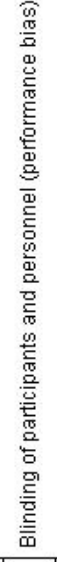 & 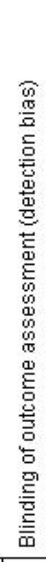 & 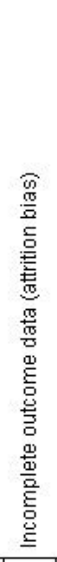 & 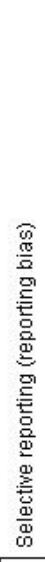 & 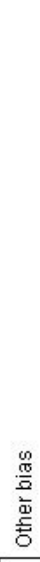 \\
\hline Ashrafinia 2015 & $\odot$ & $?$ & 0 & $?$ & $?$ & + & $?$ \\
\hline Chang 2015 & $?$ & 0 & 0 & 0 & $\odot$ & $\odot$ & $?$ \\
\hline Chen 2015 & + & 0 & 0 & 0 & $\odot$ & + & + \\
\hline Dritsa 2008 & $\odot$ & $\odot$ & 0 & $\odot$ & + & $\odot$ & $\odot$ \\
\hline Funda 2020 & $\odot$ & + & 0 & $?$ & $\odot$ & + & + \\
\hline Giallo 2014 & $\odot$ & $\odot$ & 0 & $?$ & $\odot$ & $\odot$ & $?$ \\
\hline Lee 2016 & $?$ & $?$ & O & + & + & + & $?$ \\
\hline Ozcan 2020 & $\odot$ & $\odot$ & 0 & $?$ & $\odot$ & $\odot$ & $\odot$ \\
\hline Stremler 2013 & $\odot$ & $\odot$ & 0 & $\oplus$ & $\odot$ & + & $\odot$ \\
\hline Vaziri 2017 & $\odot$ & $?$ & 0 & 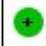 & + & $\odot$ & $?$ \\
\hline Yang 2019 & $\odot$ & † & $\theta$ & $?$ & $\odot$ & $\odot$ & $\odot$ \\
\hline
\end{tabular}

Figure 2

Risk of bias for individual RCTs

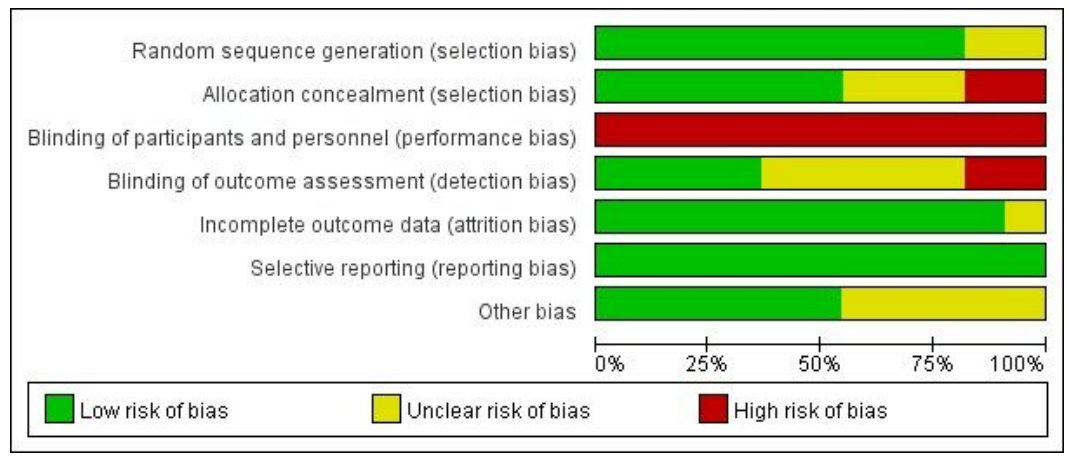

Figure 3

Risk of bias summaries for the included RCTs 


\section{A: Fatigue scores at postintervention}

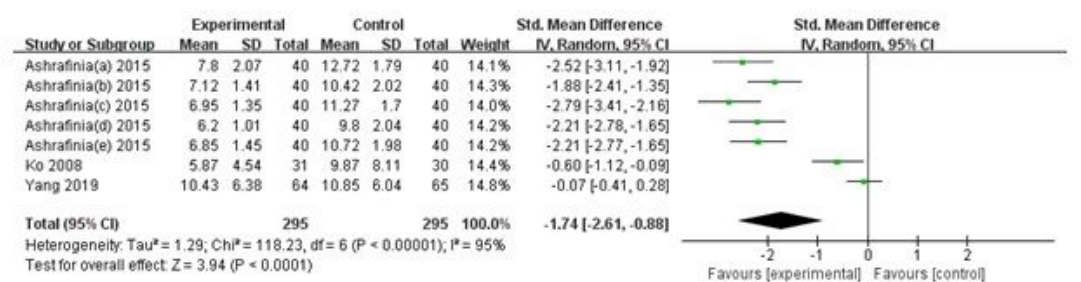

\section{B: Depression scores at postintervention}

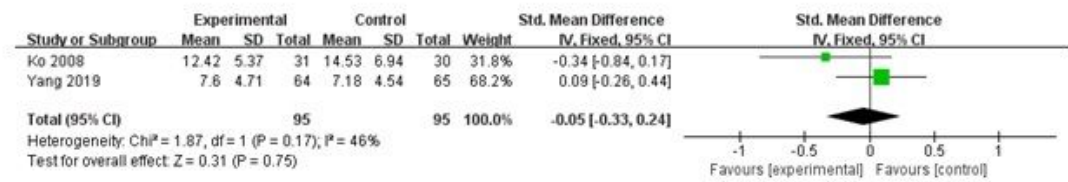

Figure 4

Forest plots for the effect of exercise on fatigue (A) and depression (B) postintervention. Notes: Ashrafinia et al.'s study assessed general fatigue (a), physical fatigue (b), reduced activity (c), reduced motivation (d), and mental fatigue (e).

\section{A: Fatigue scores at postintervention}

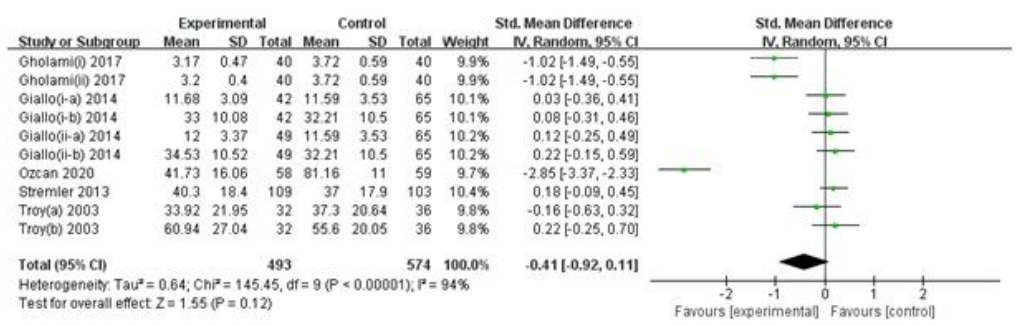

\section{B : Depression scores at postintervention}

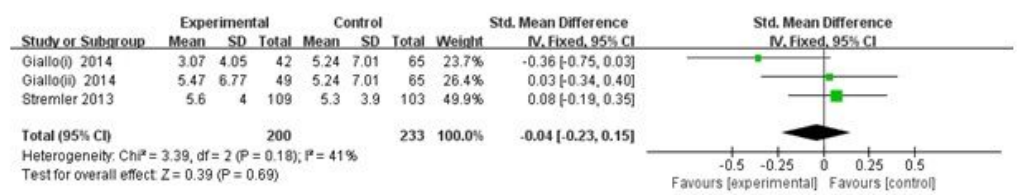

\section{C: Fatigue scores at 8-week follow-up}

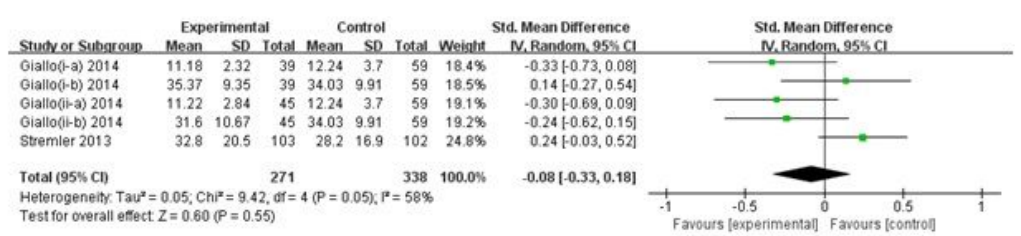

D: Depression scores at 8-week follow-up

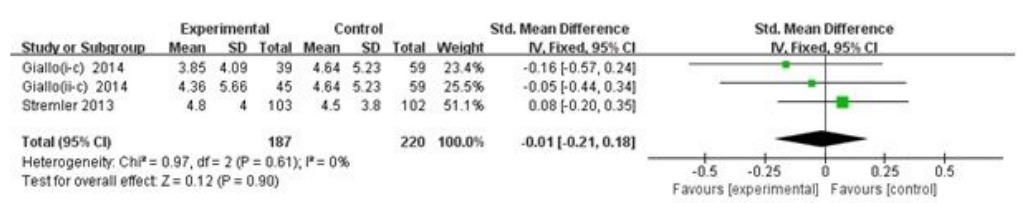

\section{Figure 5}

Forest plots for the effect of psychoeducational interventions on fatigue at postintervention (A), depression at postintervention (B), fatigue scores at the 8week follow-up (C), and depression scores at the 8-week follow-up (D). Notes: Gholami et al.'s study included two intervention groups (i: e-learning and ii: face to face), which had assessment scores. Giallo et al.'s study included two intervention groups (i: professionally led telephone support and ii: self-directed 
written), and it used two independent scales (a: FAS and b: FSS) to assess fatigue symptoms in the same group. Troy et al.'s study examined morning fatigue (a) and evening fatigue (b).

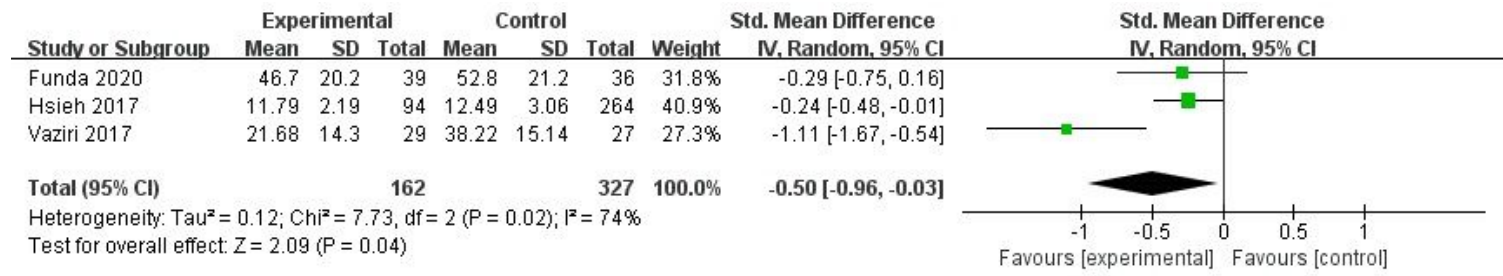

\section{Figure 6}

Forest plots for meta-analysis of physical therapy on postintervention fatigue scores.

\section{A: Fatigue scores at postintervention}

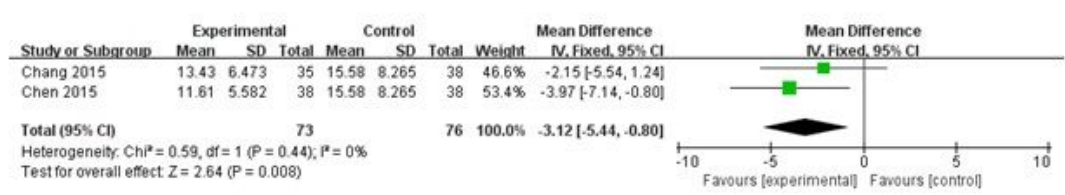

\section{B : Depression scores at postintervention}

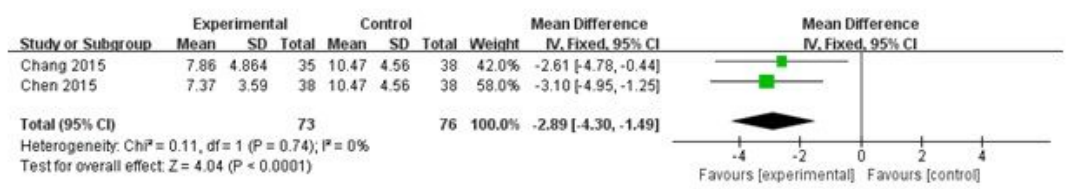

\section{C: Fatigue scores at 2-week follow-up}

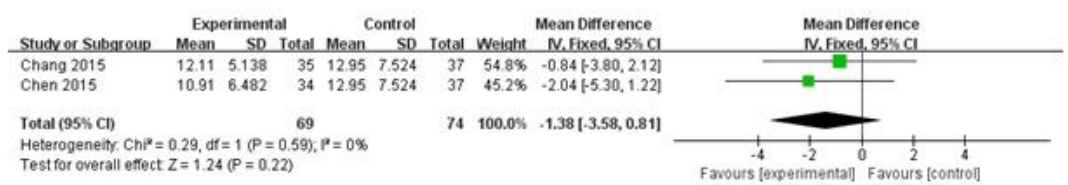

\section{D: Depression scores at 2-week follow-up}

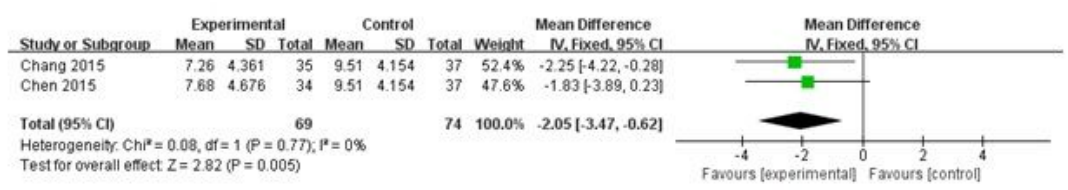

\section{Figure 7}

Forest plots for the effect of drinking tea on fatigue at postintervention (A), depression at postintervention (B), fatigue scores at the 2-week follow-up, (C) and depression scores at the 2-week follow-up (D).

\section{Supplementary Files}

This is a list of supplementary files associated with this preprint. Click to download.

- Additionalfile1PRISMA2009checklist.doc

- Additionalfile2Thefinalsearchstrategies.docx 